\title{
Initial Ten Years of Experience with the Intravitreal Dexamethasone Implant: A Retrospective Chart Review
}

This article was published in the following Dove Press journal:

Clinical Ophthalmology

\author{
Josh Wallsh' \\ Charlotte Luths $\mathbb{1 D}^{2}$ \\ Haily Kil (1D ${ }^{2}$ \\ Ron Gallemore $\mathbb{D}^{2}$
}

'Albany Medical Center, Department of Ophthalmology, Albany, NY, USA; ${ }^{2}$ Retina Macula Institute and Research Center, Torrance, CA, USA
Correspondence: Ron Gallemore Retina Macula Institute and Research Center, 420I Torrance Blvd Suite 220, Torrance, CA 90503, USA

Email rongallemoremd@gmail.com
Purpose: To evaluate the initial ten years of results from the intravitreal dexamethasone implant (DEX) in patients treated for retinal vein occlusion (RVO), diabetic macular edema (DME) or uveitis.

Methods: Retrospective chart review of patients receiving DEX since its FDA approval. Best-corrected visual acuity (BCVA), central macular thickness (CMT) on optical coherence tomography, intraocular pressure and cataract status were collected. Baseline data were collected from the initial DEX and post-treatment data at the visit at least four weeks after the last DEX.

Results: In total, 315 eyes received 1216 DEX over $63.9 \pm 4.6$ weeks. In the branch RVO $(n=90)$, central RVO $(n=59)$ and DME $(n=62)$ cohorts, BCVA improved significantly $(\mathrm{p}<0.05)$. The uveitis $(\mathrm{n}=154)$ cohort did not have a significant change in BCVA, 0.62 \pm 0.04 to $0.61 \pm 0.04 \operatorname{logMAR}(\mathrm{p}=0.34$ ). Younger patients, vitrectomized eyes, and eyes without a history of glaucoma were associated with significantly better BCVA outcomes in the uveitis cohort $(\mathrm{p}<0.05)$. Overall, CMT decreased significantly from $376.6 \pm 6.8$ to $322.7 \pm 5.0$ $\mu \mathrm{m}(\mathrm{p}<0.05)$. Intraocular pressure increased significantly $(\mathrm{p}<0.001)$ and the percentage of patients requiring anti-glaucoma medications increased from $33.0 \%$ to $67.6 \%$. Of phakic eyes, $58.8 \%(\mathrm{n}=63)$ had cataract progression or underwent surgery with those who underwent surgery experiencing a significant improvement in BCVA $(\mathrm{p}<0.05)$.

Conclusion: Repeated DEX over extended follow-up offers significant anatomic benefits to all cohorts. Visual benefits are only seen in RVO, DME and select uveitis demographics.

Keywords: uveitis, branch retinal vein occlusion, central retinal vein occlusion, diabetic macular edema, dexamethasone implant

\section{Introduction}

The intravitreal dexamethasone implant (DEX; Ozurdex; Allergan, Inc; Irvine, CA) has been Food and Drug Administration (FDA) approved for the treatment of posterior uveitis and retinal vein occlusions (RVOs) since 2009. More recently, it has also been approved for the treatment of diabetic macular edema (DME). Numerous studies examining various numbers of DEX treatments and lengths of treatment have demonstrated its effectiveness at treating $\mathrm{RVO},{ }^{1-5}$ uveitis $^{6-10}$ and DME. ${ }^{11-16}$ As patients receive multiple DEX implants over extended lengths of treatment the concern grows for more significant intraocular pressure (IOP) elevations and cataract progression, both well-documented complications. ${ }^{17-20}$ Herein, we report the initial ten years of clinical experience with the DEX implant at a busy, 
socioeconomically diverse retina practice. These realworld results allow for evaluation of the efficacy and safety of repeated DEX implants over an extended length of follow-up.

\section{Methods}

Retrospective chart review was performed of all patients at a single retina practice receiving treatment with DEX injections since FDA approval in 2009 through 2019. Patients were primarily treated for one or more of the following diseases: posterior uveitis, central RVO (CRVO), branch RVO (BRVO), and/or DME. Treatment with DEX was initiated in both treatment naïve eyes and those that were previously treated with laser, intravitreal/ periocular steroids injections or intravitreal anti-vascular endothelial growth factors (anti-VEGF) injections. In those who were previously treated, DEX was initiated due to failure to respond, inadequate response, or desire to decrease injection and/or drop burden. Failure to respond and an inadequate response to previous treatment was determined based on multiple modalities including persistent macular edema on optical coherence tomography (OCT). Determination to initiate such therapy and timing of this following prior treatment was made by the treating physician. In those eyes concurrently diagnosed with glaucoma, treatment with DEX was initiated only in those with well-controlled intraocular pressure (IOP) and, if indicated, with the assistance of the patient's glaucoma specialist. Prior to initiation of DEX treatment, patients received a complete eye examination with assessment of best-corrected visual acuity (BCVA), IOP, cataract assessment, and - when indicated - imaging with OCT (Cirrus HD-OCT, Carl Zeiss Meditec, Dublin, CA) and/or fluorescein angiography.

After the initial DEX treatment, follow-up visits were determined by the treating physician with common intervals being between four to six weeks. Similarly, the determination of further DEX treatment was made by the treating physician based on multiple assessments including BCVA and OCT response to previous DEX treatment.

Outcomes measured at baseline and final visit included BCVA, central macular thickness (CMT) on OCT, IOP and anti-glaucoma medications. When eyes were being treated by combination anti-glaucoma medications, they were counted based on the total number of pharmaceutical classes (eg, the combination drop dorzolamide-timolol would be counted as two separate medications). Baseline measurements were assessed at the visit when patients received the initial DEX implant. Final visit measurements were assessed at the visit closest to six weeks after the last DEX treatment, but at least 28 days after that treatment. Along with baseline and final visit measurements, the BCVA was also documented at the time of each DEX treatment and at follow-up visits between treatments. Measurement of CMT was performed by averaging the macular thickness of the five central subfields on OCT macular thickness maps. Adjunct treatments required between or with DEX treatment-most notably anti-VEGF injections - were documented along with performance of cataract surgery, interval between DEX treatments, total number of DEX treatments and other adverse events. For those eyes within the uveitis cohort, additional diseasespecific characteristics were documented including etiology and concurrent systemic therapy. In addition, eyes with uveitis with more than 1 year of follow-up after completion of DEX treatment were evaluated for the need for continued intravitreal or periocular injection and BCVA results.

Statistical analysis was performed comparing baseline to final visit for all outcomes using a paired Student's $t$-test as well as subset analysis of CRVO, BRVO, DME and uveitis groups.

This study was submitted to the Sterling Institutional Review Board for ethics approval as the data were collected from a location not affiliated with an institutional review board. Following review, the study was granted exempt status (IRB ID\# 8084) on the basis that patient consent was not required for this retrospective study as patient information was to be obtained and recorded in a de-identified manner. Despite this, written informed consent had previously been obtained from patients for chart review and publication. Individual health information remained protected, and data confidentiality was maintained throughout the collection of data and writing of this article according to the Health Insurance Portability and Accountability Act (HIPPA) Privacy Rule and Security Rule. The report is in adherence to the tenets of the Declaration of Helsinki.

\section{Results}

Three hundred and fifteen eyes from 270 patients received a total of 1216 DEX implants during the 10-year study. Table 1 gives the demographics for all studied cohorts and the entire study group. Within the uveitis cohort, 118 (76.6\%) eyes were previously treated; however, many of these eyes were treated with intravitreal anti-VEGF injections, focal or panretinal photocoagulation for a co-existing disease (including exudative macular degeneration, RVO, DME, etc.). For 
Table I Patient Demographics

\begin{tabular}{|c|c|c|c|c|c|}
\hline & BRVO & CRVO & Uveitis & DME & Total \\
\hline Patients & 79 & 58 & 128 & 43 & 270 \\
\hline Eyes & 90 & 59 & 154 & 62 & 315 \\
\hline \multicolumn{6}{|l|}{ Age (years) } \\
\hline Mean \pm SEM & $69.5 \pm 1.0$ & $70.1 \pm 2.0$ & $67.4 \pm 1.2$ & $68.3 \pm 1.6$ & $68.7 \pm 0.7$ \\
\hline \multicolumn{6}{|l|}{ Gender } \\
\hline Male: Female & $46: 33$ & $36: 22$ & 65: 63 & 23: 20 & I45: 125 \\
\hline \multicolumn{6}{|l|}{ Lens Status } \\
\hline Phakic: Pseudophakic & 36: 54 & $34: 25$ & 30: 124 & 18: 44 & I07: 208 \\
\hline \multicolumn{6}{|l|}{ Previously Treated Eyes* } \\
\hline Amount (\%) & 77 (85.5\%) & 54 (9l.5\%) & II8 (76.6\%) & 57 (9l.\%) & $26 \mid(82.5 \%)$ \\
\hline Intravitreal anti-VEGF & $74(82.2 \%)$ & $53(89.8 \%)$ & 89 (57.7\%) & 57 (91.\%) & $229(72.4 \%)$ \\
\hline Subtenon Kenalog & $3(3.3 \%)$ & $2(3.38 \%)$ & $50(32.4 \%)$ & $4(6.4 \%)$ & $58(18.3 \%)$ \\
\hline Intravitreal Steroid & $15(16.6 \%)$ & $9(15.2 \%)$ & $45(29.2 \%)$ & $19(30 . \%)$ & $77(24.3 \%)$ \\
\hline Focal Laser Photocoagulation & $28(31.1 \%)$ & II (I8.6\%) & 14 (9.0\%) & $31(50 \%)$ & $64(20.2 \%)$ \\
\hline Panretinal Photocoagulation & $26(28.8 \%)$ & $14(23.7 \%)$ & $14(9.0 \%)$ & $31(50 \%)$ & $63(19.9 \%)$ \\
\hline \multicolumn{6}{|l|}{ Concurrent Anti-VEGF Treatment } \\
\hline Amount (\%) & $45(50.0 \%)$ & $33(55.9 \%)$ & $54(35.1 \%)$ & $32(51.6 \%)$ & 145 (46.0\%) \\
\hline \multicolumn{6}{|l|}{ Length of Treatment (days) } \\
\hline Mean \pm SEM & $300.1 \pm 49.1$ & $442.7 \pm 78.4$ & $565.4 \pm 52.5$ & $247.4 \pm 35.30$ & $447.4 \pm 32.3$ \\
\hline Total DEX Injections & 247 & 206 & 744 & 153 & 1216 \\
\hline
\end{tabular}

Note: *Treatments included intravitreal anti-VEGF injections, steroid injections, focal and panretinal photocoagulation.

Abbreviations: SEM, standard error of means; DEX, intravitreal dexamethasone implants; BRVO, branch retinal vein occlusion; CRVO, central retinal vein occlusion; DME, diabetic macular edema.

all eyes that received previous anti-VEGF, subtenon Kenalog, intravitreal steroids, focal and panretinal photocoagulation an average of $7.5 \pm 0.7,2.8 \pm 0.2,3.0 \pm 0.3,2.0 \pm$ 0.1 , and $1.8 \pm 0.1$ treatments were performed, respectively. These averages were relatively consistent across all cohorts. Forty-two eyes had multiple diagnoses in their treated eye: 33 - BRVO and DME; 4 - uveitis and DME; 6 - uveitis and CRVO; 3 - uveitis and BRVO; 2 - CRVO and DME; and 1 uveitis, BRVO, and DME. Of eyes receiving multiple DEX treatments, the average interval between injections was 126.0 \pm 5.1 days. Numerous eyes discontinued treatment with DEX and restarted 1 or more years later, excluding all treatment intervals $>365$ days resulted in an average treatment interval of $103.6 \pm 2.0$ days. Subgroup analysis revealed similar treatment intervals within each cohort.

\section{Retinal Vein Occlusions and Diabetic Macular Edema}

The CRVO, BRVO, and DME cohorts all experienced statistically significant improvements in BCVA and
CMT. Visual acuity improved from $0.97 \pm 0.08$ to 0.88 $\pm 0.07 \log$ MAR in the CRVO cohort, $0.76 \pm 0.06$ to 0.66 $\pm 0.06 \log \mathrm{MAR}$ in the BRVO cohort, and $0.73 \pm 0.06$ to $0.61 \pm 0.06 \operatorname{logMAR}$ in the DME cohort (Figure 1; $<<$ 0.05). Central macular thickness improved significantly from $355.1 \pm 15.1$ to $308.0 \pm 13.1 \mu \mathrm{m}$ in the CRVO cohort, $399.6 \pm 13.8$ to $337.9 \pm 9.3 \mu \mathrm{m}$ in the BRVO cohort, and $428.4 \pm 23.3$ to $320.1 \pm 10.7 \mu \mathrm{m}$ in the DME cohort (Figure 2; $<<0.05$ ). Evaluation of the change in BCVA compared to baseline following each DEX treatment demonstrates a trend of improving vision with more injections (Figure 3). The sample size diminishes significantly with increasing injections resulting in less consistent data. In addition, the BRVO graph has a notable large increase after the first treatment which was in part due to multiple eyes that had significant cataract progression requiring subsequent surgery.

Comparing eyes with DME from baseline to final visit based on lens status (pseudophakic throughout, phakic throughout, underwent cataract surgery during study) 


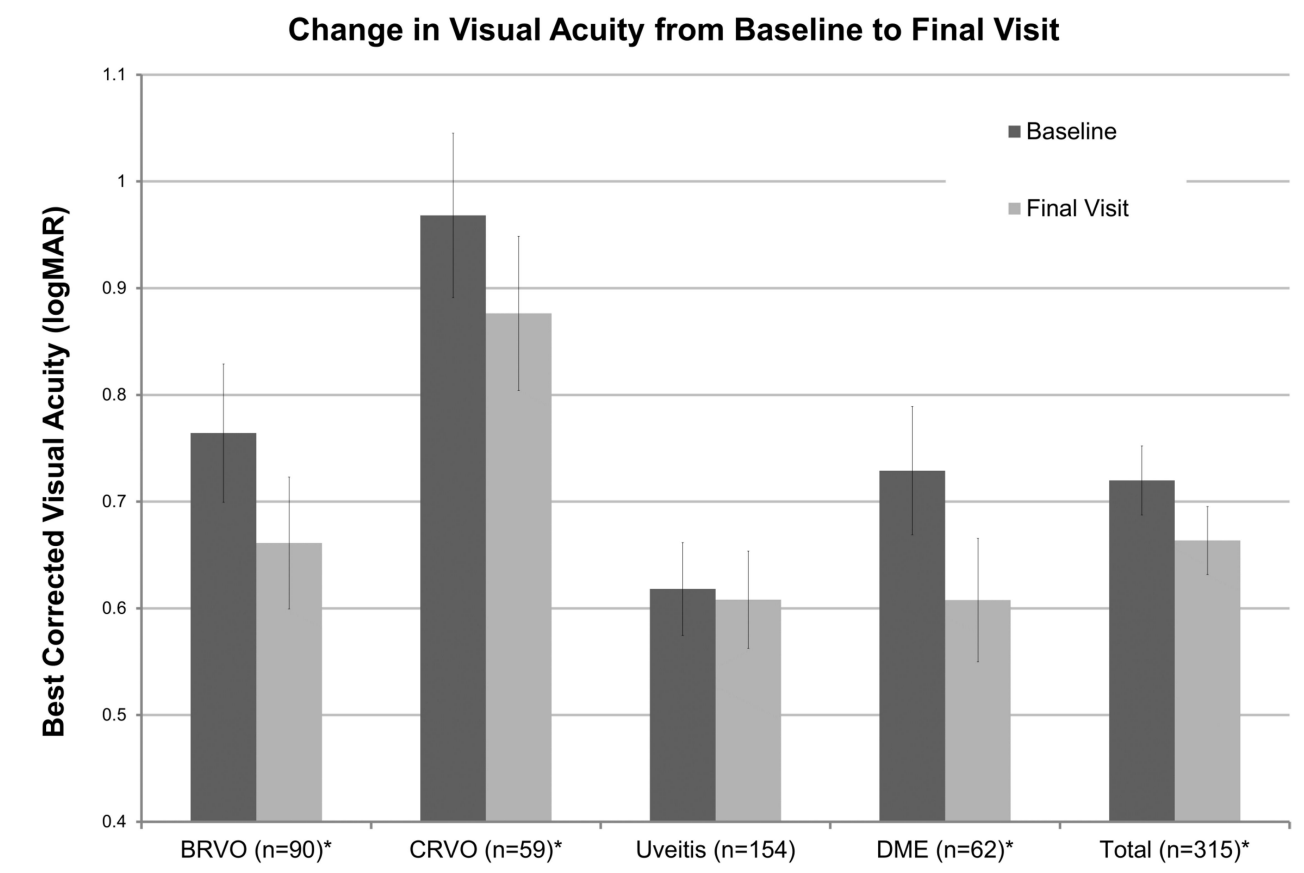

Figure I Comparison of best-corrected visual acuity from baseline to final visit for each cohort and all studied eyes. *Statistically significant ( $\mathrm{P}<0.05$ ). Abbreviations: BRVO, branch retinal vein occlusion; CRVO, central retinal vein occlusion; DME, diabetic macular edema.

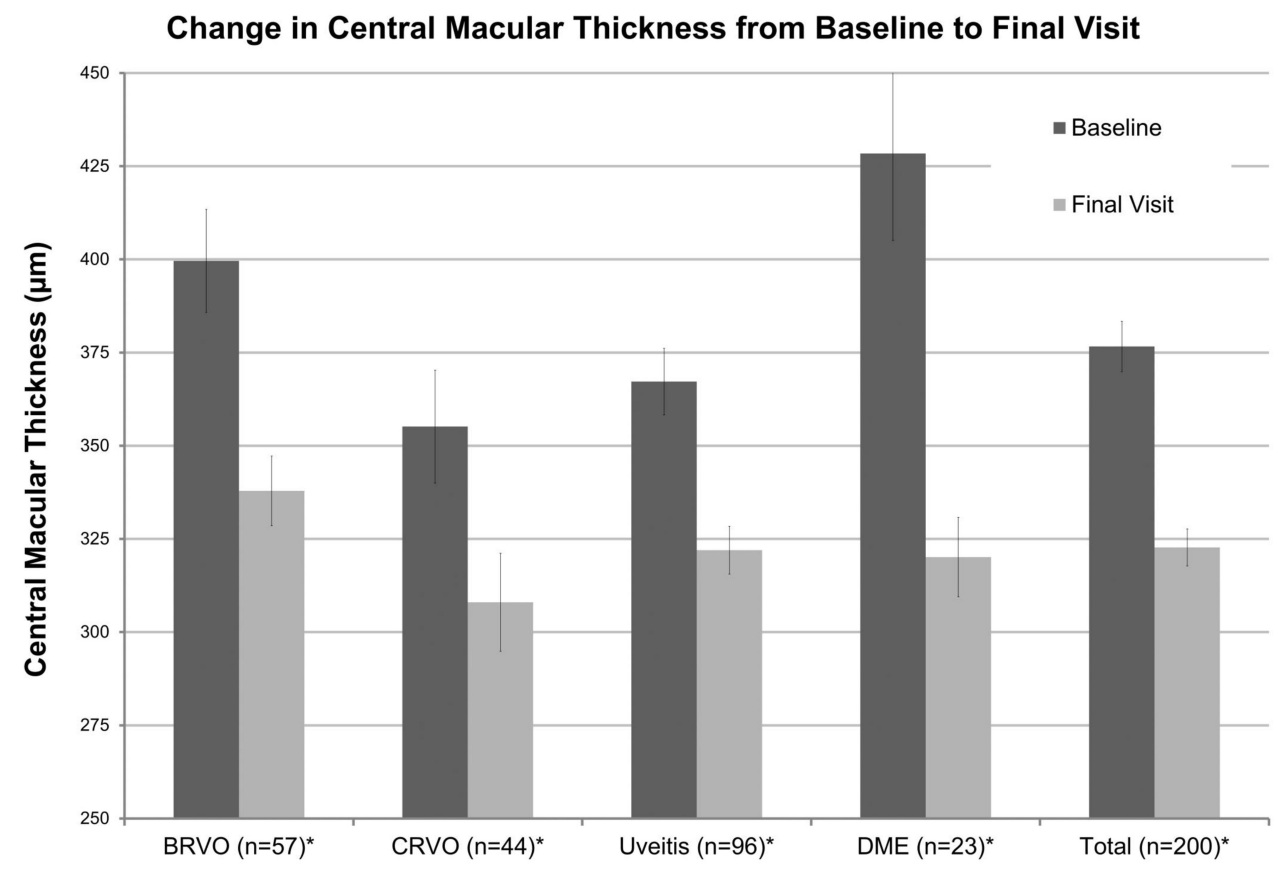

Figure 2 Comparison of central macular thickness on optical coherence tomography (OCT) from baseline to final visit for each cohort and all studied eyes. * Statistically significant $(p<0.05)$.

Abbreviations: BRVO, branch retinal vein occlusion; CRVO, central retinal vein occlusion; DME, diabetic macular edema.

demonstrated comparable improvements in BCVA and CMT for all subgroups. Further statistical analysis of the average change in BCVA and CMT between subgroups revealed nonsignificant differences between all subgroups except for the CMT in pseudophakic eyes compared to those who underwent cataract surgery (Figure 4; $<<0.05$ ). 

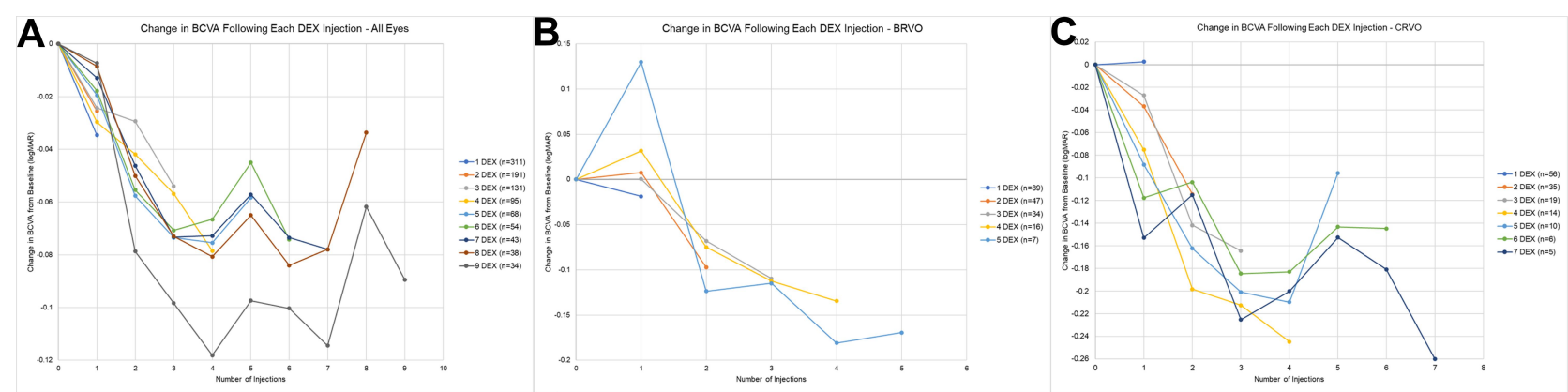

$\mathbf{D}_{0}$

Change in BCVA Following Each DEX Injection - DME

$E$
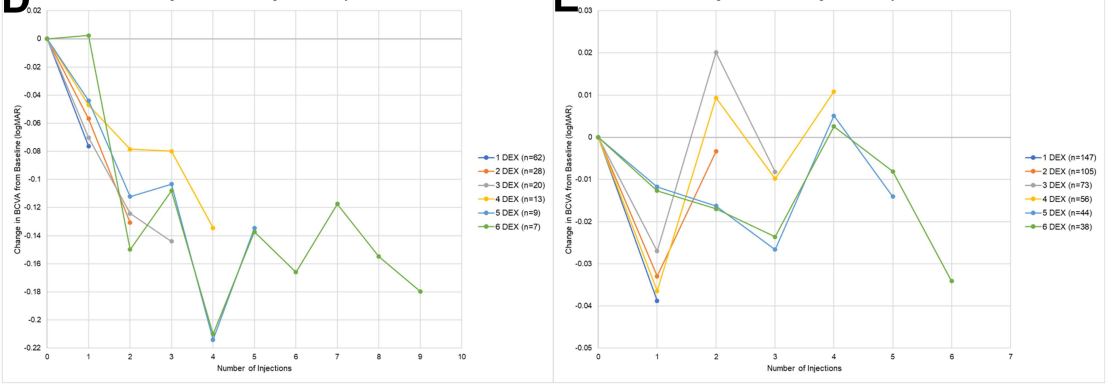

Figure 3 Change in BCVA compared to baseline following each DEX injection. Each graphed line includes only those patients who received at least the amount of DEX injections noted in the legend. Decreasing values signify improvement in BCVA. The BCVA values were recorded preferentially at the follow-up visit closest to six weeks after DEX injection and at least four weeks after treatment date. (A) All studied eyes, (B) BRVO, (C) CRVO, (D) DME, and (E) uveitis.

Abbreviations: BCVA, best-corrected visual acuity; BRVO, branch retinal vein occlusion; CRVO, central retinal vein occlusion; DME, diabetic macular edema.

600

DME Change from Baseline to Final Visit Based on Lens Status

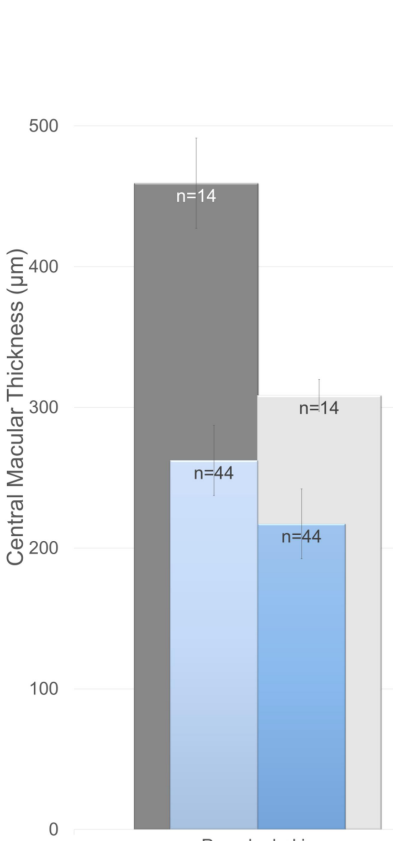

Pseudophakic

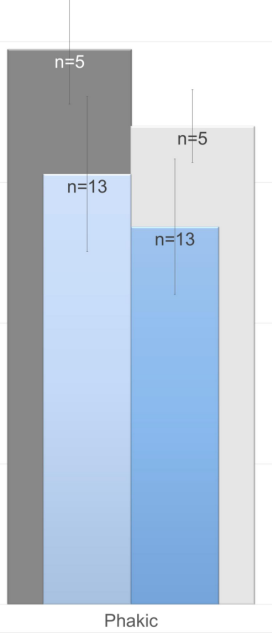

$\sqcup$ Final Visit (CMT)
- Basline (CMT)

$\because$ Baseline (BCVA)

$\uplus$ Final Visit (BCVA)

1.4

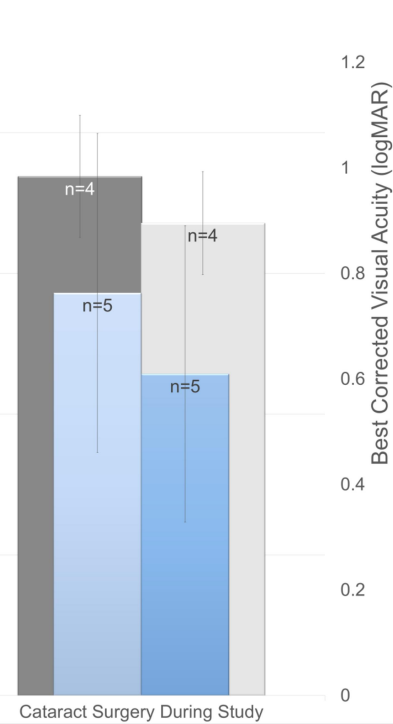

Figure 4 Comparison of baseline and final visit BCVA and CMT results for DME cohort based on lens status (pseudophakic throughout, phakic throughout, or underwent cataract surgery during study). Those eyes that underwent cataract surgery did so after an average of $1.6 \pm 0.2$ DEX implants. The average change in BCVA and CMT was compared across each subgroup utilizing a Student's t-test. Based on BCVA the following p-values were calculated: pseudophakic vs phakic -0.60 , pseudophakic vs cataract surgery -0.83 , and phakic vs cataract surgery -0.62 . For CMT data the following p-values were calculated: pseudophakic vs phakic -0.09 , pseudophakic vs cataract surgery 0.04 , phakic vs cataract surgery 0.62 .

Abbreviations: BCVA, best-corrected visual acuity; CMT, central macular thickness; DEX, intravitreal dexamethasone implant. 


\section{Uveitis}

The uveitis cohort experienced a nonsignificant improvement in BCVA from $0.62 \pm 0.04$ to $0.61 \pm 0.04 \operatorname{logMAR}(\mathrm{p}$ $=0.34$, Figure 1). Similar to these findings, Figure 3 demonstrates that the change in BCVA following each injection in the uveitis cohort minimally fluctuates around 0 between -0.04 and $0.02 \log$ MAR. Despite this, CMT improved significantly from $367.2 \pm 8.9$ to $322.0 \pm 6.4 \mu \mathrm{m}$ ( $p<0.05$, Figure 2). Subgroup analysis of the change in BCVA from baseline to final visit was performed to compare various characteristics. These results demonstrated significantly improved BCVA outcomes in the vitrectomized eyes, eyes without glaucoma diagnosis and age $<60$ years old $(\mathrm{p}<0.05$; Figure 5). Most eyes were diagnosed with idiopathic posterior uveitis $(\mathrm{n}=135)$ with $8(5.1 \%)$ and $12(7.7 \%)$ eyes concurrently being treated with systemic steroids or immunomodulatory therapy, respectively (Table 2). Of eyes with at least 1 year of follow-up after completion of DEX therapy, 39 (41.0\%) eyes required no further periocular or intravitreal treatment. These eyes experienced a statistically significant decrease in BCVA during this follow-up; however, 4 eyes developed profound vision loss due to primary openangle glaucoma unrelated to uveitis or steroid therapy. Excluding these eyes reveals a nonsignificant decrease from $0.37 \pm 0.06$ to $0.41 \pm 0.08(\mathrm{p}=0.19)$.

\section{Complications}

The most prominent complication was cataract progression with documented progression or cataract surgery in $58.8 \%$ of phakic eyes. Of those patients, 46 eyes underwent cataract surgery with a significant improvement in BCVA from baseline, $0.66 \pm 0.09 \log \mathrm{MAR}$, to final visit, $0.55 \pm$ $0.08 \log$ MAR $(\mathrm{p}<0.05$, Figure 6$)$.

Intraocular pressure increased to $>25 \mathrm{mmHg}$ in 34 $(10.8 \%)$ eyes with most successfully managed medically; however, $22(6.9 \%)$ eyes required incisional glaucoma surgery. The most common diagnosis in those eyes

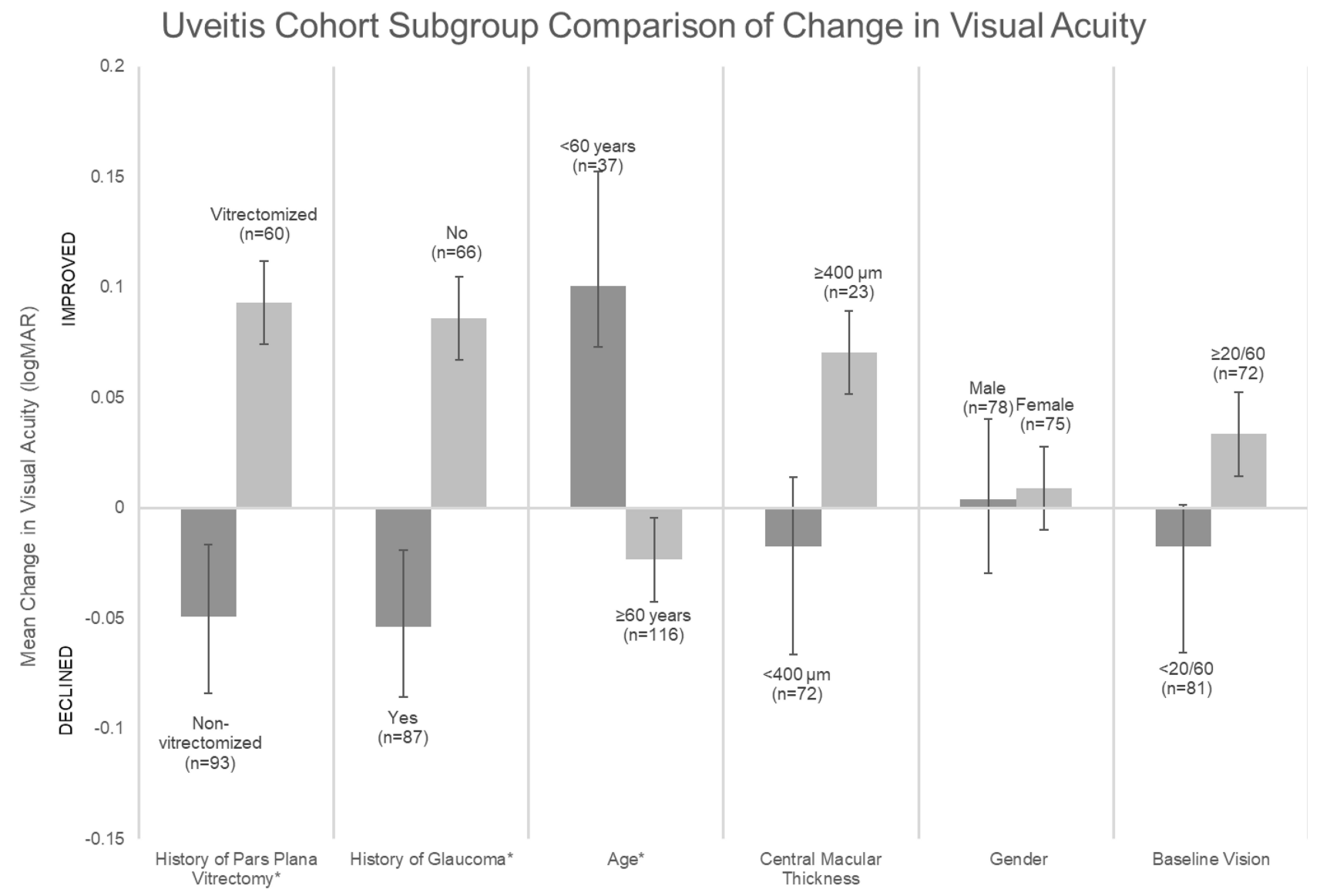

Figure 5 Uveitis cohort subgroup analysis with evaluation of the change in BCVA for various patient characteristics. Improvement in BCVA noted for the vitrectomized eyes, eyes without glaucoma diagnosis, age less than 60 years old, CMT greater than or equal to $400 \mu \mathrm{m}$ and baseline visual acuity greater than or equal to $20 / 60 . *$ Statistically significant $(p<0.05)$.

Abbreviations: BCVA, best-corrected visual acuity; CMT, central macular thickness. 
Table 2 Uveitis Cohort Supplemental Information

\begin{tabular}{|l|l|}
\hline Etiology & Eyes (Percent) \\
\hline Idiopathic & $135(87.6 \%)$ \\
Birdshot & $7(4.5 \%)$ \\
Retinitis Pigmentosa & $7(4.5 \%)$ \\
HLA-B27 & $2(1.2 \%)$ \\
Vogt-Koyanagi-Harada & $2(1.2 \%)$ \\
Psoriasis & $1(0.6 \%)$ \\
\hline Concurrent Oral Steroid Therapy & Eyes (Percent) \\
\hline Yes & $8(5.1 \%)$ \\
\hline No & $146(94.8 \%)$ \\
\hline Concurrent Immunomodulatory Therapy & Eyes (Percent) \\
\hline Yes & $12(7.7 \%)$ \\
No & $142(92.2 \%)$ \\
\hline Eyes with >I Year of Follow-up After Completing DEX Treatment \\
(n=95) & Eyes (Percent) \\
\hline Intravitreal or Periocular Injection During & \\
Follow-up & $58(61.0 \%)$ \\
\hline Yes & $37(38.9 \%)$ \\
\hline No & Mean \pm SEM \\
\hline Eyes without Additional Injections (n=39) & $0.47 \pm 0.08$ \\
\hline Length of Follow-up (years) & $0.59 \pm 0.1 I^{*}$ \\
\hline BCVA at DEX Treatment Completion & \\
\hline BCVA at Most Recent Follow-up & No.3 \\
\hline
\end{tabular}

Note: *Compared to DEX Treatment Completion ( $p<0.05)$.

Abbreviations: DEX, intravitreal dexamethasone implant; BCVA, best-corrected visual acuity.

requiring incisional glaucoma surgery was steroid-induced glaucoma $(\mathrm{n}=13)$ followed by uveitic glaucoma $(\mathrm{n}=5)$, mixed mechanism with primary open-angle and steroidinduced glaucoma $(\mathrm{n}=2)$, primary open-angle glaucoma $(\mathrm{n}=1)$, and neovascular glaucoma $(\mathrm{n}=1)$. No other complication occurred in more than $1 \%$ of eyes (Table 3). On average, eyes reached their maximum IOP after $2.1 \pm 0.1 \mathrm{DEX}$ treatments. Sixty-eight $(21.6 \%)$ eyes were noted to have a maximum IOP $\geq 10 \mathrm{mmHg}$ above baseline or maximum IOP $>25 \mathrm{mmHg}$. Evaluating only these eyes demonstrated that the majority of these eyes reached this after less than 3 injections and less than 6 months (Table 4). The amount of anti-glaucoma medications being used significantly increased from $0.71 \pm 0.06$ to $1.50 \pm 0.07$ drops with 105 (33.0\%) eyes using anti-glaucoma medications at baseline and 213 (67.6\%) eyes at the final visit. Overall, $133(42.2 \%)$ eyes required an additional or initial anti-glaucoma medication following DEX treatment.

\section{Discussion}

In this study, we retrospectively examine the results of 10 years of experience with the intravitreal dexamethasone implant. In total, the studied eyes were treated with an average of 3.9 implants every 3.4 months witnessing an improvement in visual acuity and central macular thickness.

\section{Retinal Vein Occlusions and Diabetic Macular Edema}

The benefits of a single DEX implant in patients with $\mathrm{RVOs}^{2,4}$ or DME ${ }^{12,13}$ have been demonstrated numerous times with similar benefits and safety profile noted in the use of multiple DEX implants over an extended time period. ${ }^{1,5,11,14,15,19,21,22}$ Two of the larger studies evaluating multiple DEX treatments in RVO patients found somewhat differing results. SHASTA evaluated at least 2 DEX implants in RVO eyes and noted significant improvements in BCVA and macular thickening over the 1.2-year trial. ${ }^{5}$ Busch et al followed RVO patients for 2 years after initial DEX treatment and noted nonsignificant BCVA changes over that time frame. However, at the 1-year mark, significant improvements in BCVA were noted in both the BRVO and CRVO group and macular thickening was noted to improve significantly at the 2-year mark. Only a small percentage of these patients were pseudophakic $(17.0 \%)$ or underwent cataract surgery during the study (27.6\% of phakic eyes) and since cataract progression was not reported it is possible that lens changes are to blame for the BCVA decline at 2 years. ${ }^{22}$ In our study, we noted statistically significant improvements in BCVA and CMT in both the BRVO and CRVO cohorts at 300.1 \pm 49.1 and $442.7 \pm 78.4$ days, respectively. These are consistent with both of the aforementioned studies at the 1-year time frame.

Regarding those eyes with DME, the 62 studied eyes were also noted to have a statistically significant improvement in BCVA and CMT over the studied period. Again, these results in eyes with DME are consistent with previously published literature regarding DME treated with multiple DEX treatments. ${ }^{11,16,23,24}$ Despite the revised indication by the FDA for DME to include phakic eyes the decision to begin DEX therapy in such eyes remains controversial. Multiple studies have demonstrated robust improvements in both BCVA and CMT while treating phakic eyes with many eventually requiring cataract surgery for optimal visual outcomes. ${ }^{11,23,24}$ Our study 


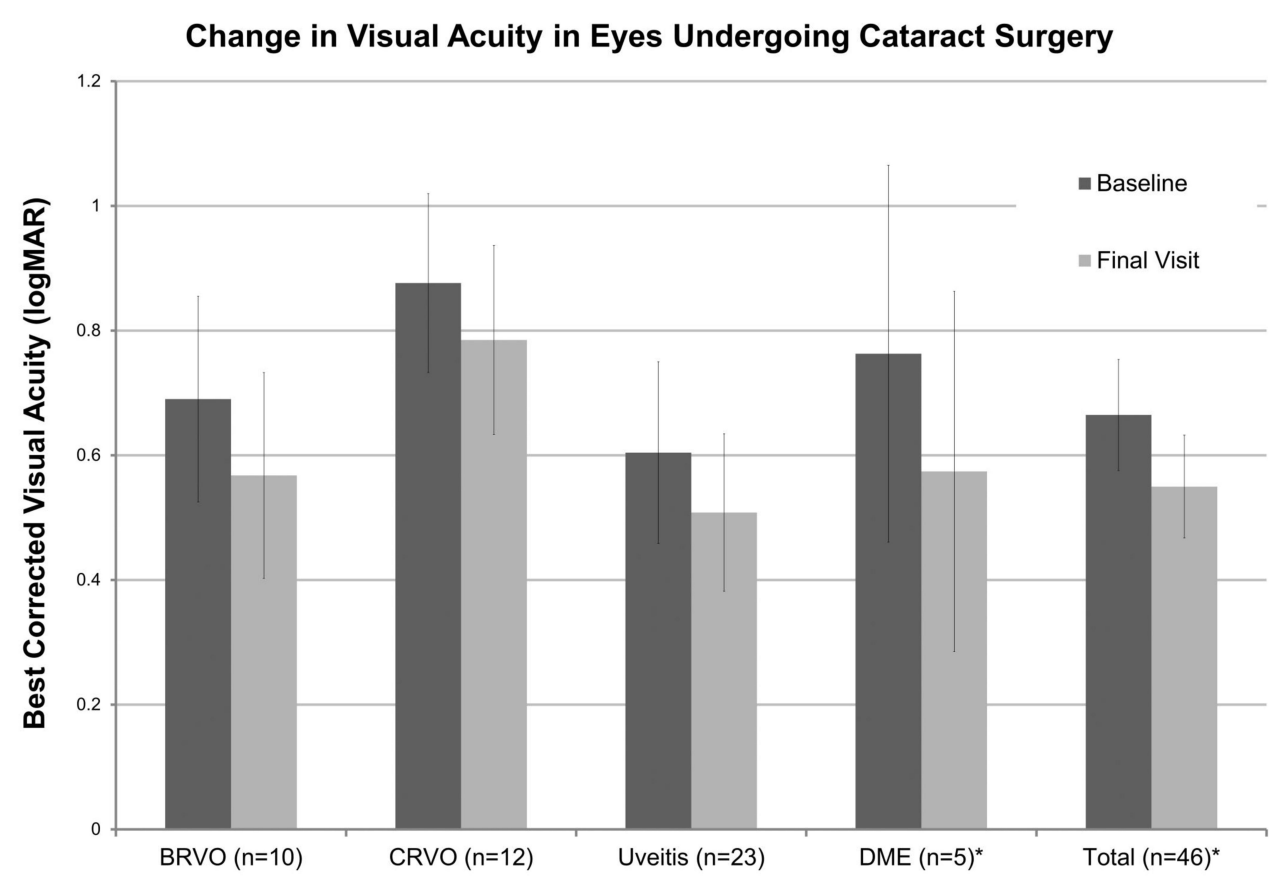

Figure 6 Best-corrected visual acuity in eyes undergoing cataract surgery compared from baseline (prior to initiation of intravitreal dexamethasone implant treatment) to the final visit. *Statistically significant $(p<0.05)$.

Abbreviations: BRVO, branch retinal vein occlusion; CRVO, central retinal vein occlusion; DME, diabetic macular edema.

population appreciated similar improvements in both BCVA and CMT over the study course whether they were pseudophakic or phakic at initiation of the study. It is important to note that these results are limited by the small sample size of only 18 total phakic eyes with DME at initiation.

Given the various lengths of follow-up and amounts of DEX implants in our study population, evaluation of baseline to final visit BCVA does not fully reveal the impact of multiple DEX implants. However, when evaluating the change in BCVA against number of DEX implants a consistent trend of improvement with escalating DEX implants can be appreciated in the BRVO, CRVO and DME cohorts. An important limitation to be aware of with this data is the small sample size as injection amounts increase which is especially pronounced for those receiving 4 or more DEX treatments.

\section{Uveitis}

In the treatment of uveitis, the DEX implant has routinely demonstrated substantial anatomic improvement in the form of decreased macular thickness, but some studies demonstrate more modest improvements in BCVA..$^{8,19,25}$ Our uveitis cohort of 154 eyes represents the largest studied population and while they experienced a statistically significant improvement in CMT $(p<0.05)$ their BCVA, on average, improved only slightly $(\mathrm{p}=0.34)$. Interestingly, Rajesh et al published on a similarly large cohort of eyes with uveitis $(n=149)$ and noted a slight decrease in the BCVA. ${ }^{19}$ To better elucidate whether certain factors were associated with improved BCVA results we performed subgroup analysis comparing change in BCVA from baseline to final visit. Those characteristics that were associated with significantly improved BCVA outcomes include a history of vitrectomy, absence of glaucoma, and age less than 60 years old $(\mathrm{p}<0.05)$. Younger eyes have a better visual response to the DEX treatment which is not surprising as multiple previous studies have demonstrated similar diminishment in response in older eyes. ${ }^{26,27}$ Likely a contributing factor for the diminished response in older populations is the chronicity of their illness making it more recalcitrant to therapy.

Surprisingly, those eyes that had previously undergone vitrectomy were found to have improved BCVA results. Multiple studies have demonstrated at least equivalence in BCVA response to DEX in vitrectomized versus nonvitrectomized eyes. ${ }^{28,29}$ These results were in eyes with DME; however, in our cohort of eyes with uveitis, a significantly better BCVA outcome was noted in the vitrectomized eyes. The majority of these vitrectomized eyes were treated with membrane peeling procedures at the time of vitrectomy for epiretinal membrane or macular 
Table 3 Complications

\begin{tabular}{|c|c|c|c|c|c|}
\hline Intraocular Pressure & BRVO & CRVO & Uveitis & DME & Total \\
\hline \multicolumn{6}{|l|}{ Baseline $(\mathrm{mmHg})$} \\
\hline Mean \pm SEM & $14.4 \pm 0.5$ & $15.0 \pm 0.5$ & $14.7 \pm 0.3$ & $15.1 \pm 0.7$ & $14.7 \pm 0.2$ \\
\hline \multicolumn{6}{|l|}{ Final Visit $(\mathrm{mmHg})$} \\
\hline Mean \pm SEM & $16.0 \pm 0.4$ & $16.0 \pm 0.8$ & $15.3 \pm 0.4$ & $16.3 \pm 0.6$ & $15.8 \pm 0.2$ \\
\hline Lens Change in Phakic Patients & BRVO & CRVO & Uveitis & DME & Total \\
\hline \multicolumn{6}{|l|}{ Cataract Progression } \\
\hline Amount (\% of Phakic Patients) & $6(16.6 \%)$ & 9 (26.4\%) & $2(6.6 \%)$ & $4(22.2 \%)$ & $17(15.8 \%)$ \\
\hline \multicolumn{6}{|l|}{ Cataract Surgery } \\
\hline Amount (\% of Phakic Patients) & $10(27.7 \%)$ & $12(35.2 \%)$ & $23(79.3 \%)$ & $5(27.7 \%)$ & $46(42.9 \%)$ \\
\hline \multicolumn{6}{|l|}{ Cataract Surgery or Progression } \\
\hline Amount (\% of Phakic Patients) & $16(44.4 \%)$ & 21 (6I.7\%) & $25(83.3 \%)$ & $9(50 \%)$ & $63(58.8 \%)$ \\
\hline Other Complications & BRVO & CRVO & Uveitis & DME & Total \\
\hline \multicolumn{6}{|l|}{ Incisional Glaucoma Surgery } \\
\hline Amount (\%) & I (I.II\%) & $3(5.08 \%)$ & $18(11.6 \%)$ & $0(0 \%)$ & $22(6.9 \%)$ \\
\hline \multicolumn{6}{|l|}{ Anterior Chamber DEX Migration } \\
\hline Amount (\%) & $0(0 \%)$ & $0(0 \%)$ & $2(1.30 \%)$ & $0(0 \%)$ & $2(0.6 \%)$ \\
\hline \multicolumn{6}{|l|}{ Endophthalmitis } \\
\hline Amount (\%) & $0(0 \%)$ & $0(0 \%)$ & 3 (1.96\%) & $0(0 \%)$ & $3(0.9 \%)$ \\
\hline \multicolumn{6}{|l|}{ Vitreous Hemorrhage } \\
\hline Amount (\%) & $0(0 \%)$ & I (1.69\%) & I (0.65\%) & $0(0 \%)$ & $2(0.6 \%)$ \\
\hline
\end{tabular}

Abbreviations: SEM, standard error of means; BRVO, branch retinal vein occlusion; CRVO, central retinal vein occlusion; DME, diabetic macular edema; DEX, intravitreal dexamethasone implant.

hole. Development of epiretinal membranes is a common occurrence in uveitis and may play a role in the diminished BCVA outcomes in non-vitrectomized eyes.

By definition, all uveitis eyes had chronic conditions as this is the only FDA approved indication for DEX in eyes with uveitis. As such, permanent damage from cystoid macular edema and glaucoma may contribute to poor visual results. The subgroup analysis discussed above revealed that those eyes diagnosed with glaucoma had significantly worse BCVA outcomes than those without glaucoma which is consistent with the notion of permanent damage from co-existing chronic illnesses. In addition, the progression from cataracts can also be a contributing factor in these eyes' visual outcomes. Cataract progression was noted in 25 uveitis eyes with 23 undergoing cataract surgery during the studied period. Those 23 eyes who underwent cataract surgery experienced an improvement in BCVA from $0.60 \pm 0.15$ to 0.51 $\pm 0.13 \log$ MAR pre- and post-cataract surgery $(\mathrm{p}=0.14)$. Despite not reaching statistical significance, this improvement demonstrates that the cataracts are contributing to these patients poor BCVA results. This chronic condition and its associated complications represent a difficult to manage entity requiring a multimodal approach. The presented results demonstrate that DEX is an integral part of this treatment protocol, but there should be thought regarding patient selection for best outcomes.

While these results demonstrate the benefits of DEX when actively being treated, they do not offer insight into the response when such treatment is discontinued. Ninetyfive eyes had more than 1 year of follow-up after completing treatment with DEX with 39 eyes requiring no further intravitreal or periocular injections. Over $4.6 \pm 0.3$ years of follow-up, these 39 eyes had a significant decrease in BCVA from approximately 20/60 to 20/80. However, 4 of these eyes had profound vision loss due to glaucomatous damage unrelated to DEX treatment or uveitis during this follow-up. Overall, these results demonstrate that many eyes treated with DEX can have many years of relative quiescence associated with such treatment.

\section{Complications}

Cataract progression and IOP elevations are welldocumented complications in literature. Prior studies of repeated DEX implants have reported varying degrees of 
Table 4 Maximum Intraocular Pressure

\begin{tabular}{|c|c|}
\hline \multicolumn{2}{|l|}{ All Eyes } \\
\hline Maximum IOP & \\
\hline Mean \pm SEM & $19.8 \pm 0.3$ \\
\hline Number of DEX to Maximum IOP & \\
\hline Mean \pm SEM & $2.1 \pm 0.1$ \\
\hline Time to Maximum IOP (days) & \\
\hline Mean \pm SEM & $224.51 \pm 20.9$ \\
\hline \multicolumn{2}{|c|}{ Eyes with Max IOP $>25 \mathrm{mmHg}$ or Change $\geq 10 \mathrm{mmHg}(\mathrm{n}=68)$} \\
\hline Number of DEX to Maximum IOP & \\
\hline Mean \pm SEM & $3.2 \pm 0.5$ \\
\hline Time to Maximum IOP (days) & \\
\hline Mean \pm SEM & $347.6 \pm 63.5$ \\
\hline Maximum IOP After I DEX & \\
\hline Amount (\% of Eyes) & $34(50.0 \%)$ \\
\hline Maximum IOP After $\leq 2 \mathrm{DEX}$ & \\
\hline Amount (\%) & $45(66.1 \%)$ \\
\hline Maximum IOP After $\leq 3 \mathrm{DEX}$ & \\
\hline Amount (\%) & $55(80.8 \%)$ \\
\hline Maximum IOP After $\leq 6$ Months & \\
\hline Amount (\%) & $34(50 \%)$ \\
\hline
\end{tabular}

Abbreviations: SEM, standard error of means; IOP, intraocular pressure; DEX, intravitreal dexamethasone implant.

cataract progression from $30 \%$ to $69 \%$. $^{1,3,11,14,19,21,30}$ Despite the repeated DEX treatment and extended follow-up, phakic eyes $(n=107)$ in our study experienced similar rates as those previously reported with $58.8 \%$ having cataract progression or requiring cataract surgery. While this should be a consideration in treating phakic eyes with DEX, it should not serve as a contraindication as cataract surgery offers a solution in those with visually significant cataract development. Evaluation of those eyes in our study who underwent cataract surgery revealed a statistically significant improvement in BCVA from baseline to final visit. This supports the notion that cataract progression may be an overly emphasized complication and concern when considering DEX treatment.

In our study population, $21.6 \%$ of eyes experienced a maximum IOP $>25 \mathrm{mmHg}$ and/or reached a maximum IOP $\geq 10 \mathrm{mmHg}$ above baseline, which was a similar rate as reported in other studies. ${ }^{17,19,31-33}$ However, $42.2 \%$ of eyes required the addition of at least one anti-glaucoma medication, either initiating or escalating therapy. This is slightly higher than the $29.1 \%$ in SHASTA, ${ }^{5} 31 \%$ reported by SAFODEX, ${ }^{31}$ and $35.8 \%$ reported by Haller et al. ${ }^{3}$ Many of the eyes initiating such therapy in our study were doing so as adjuvant treatment for macular edema rather than elevated IOP. We have previously reported the efficacy of carbonic anhydrase inhibitors in the treatment of cystoid macular edema $^{34}$ and similar results have been published for antiglaucoma medications in the treatment of DME. ${ }^{35}$ Another important factor is the higher rate of anti-glaucoma medication usage at baseline in our study cohort than previously reported, 33.0\%. Rajesh et al reported the largest cohort of DEX treatments (6015 implants). This study population only included $4.8 \%$ of eyes treated with anti-glaucoma medications at baseline. ${ }^{19}$ The safe usage of DEX in these higher risk eyes supports its use in patients with well-controlled glaucoma and ocular hypertension.

Within our study group, the rate of surgical intervention for glaucoma was significantly higher than in any previous study with $6.9 \%$ of eyes requiring incisional surgery compared to reported rates of incisional surgical intervention of 0-1.9\%. ${ }^{10,11,18,19,31}$ Evaluating these numbers further reveals that a significant majority were in the uveitis cohort $(n=18)$. Many of these eyes were undergoing incisional glaucoma surgery for documented uveitic glaucoma $(n=5)$, but given the large number of eyes within the uveitis cohort more of these eyes may have had an inflammatory component to their recalcitrant glaucoma. In a smaller study, Adán et al similarly reported a higher rate of incisional glaucoma surgery in eyes with uveitis, $5.9 \% .^{36}$ The co-administration of intravitreal anti-VEGF injections in many studied eyes may have also resulted in an increased prevalence of glaucomatous changes. Du et al reported a significantly increased rate of glaucoma procedures in eyes treated with repeated intravitreal antiVEGF injections. ${ }^{37}$ Further evaluation of the impact of combination DEX and anti-VEGF injections on glaucoma development and progression appears to be warranted.

Previous investigations into the time to reach maximum IOP have demonstrated that these responses are usually apparent earlier in the treatment process. Maturi et al reported $78 \%$ of eyes with maximum IOP $\geq 10 \mathrm{mmHg}$ above baseline occurred after the 1 st or 2 nd DEX treatment. ${ }^{32}$ Our study population demonstrated similar results with $80.8 \%$ of eyes with maximum IOP $>25 \mathrm{mmHg}$ or change in IOP $\geq 10 \mathrm{mmHg}$ above baseline reaching their peak after the 1st, 2nd, or 3rd DEX. Based on these results, eyes whose IOP will respond to steroids will generally do so after the first few DEX treatments. However, 6 studied eyes reached this IOP mark following 10 or more injections $(10,10,12,15,25$ and 25 DEX treatments) so it is important to remain vigilant about the possibility of a steroid response after every DEX treatment.

Other complications such as vitreous hemorrhage, endophthalmitis, AC migration occurred at a much lower 
rate (under $1 \%$ ). Our results support the safety profile of DEX from previous literature and demonstrate that this continues with many years of repeated treatment. The risk of cataract progression and IOP elevations are significant with DEX treatment, but patients overall respond well to medical and surgical management options of these complications. As such, the possibility of these complications should not serve as absolute contraindications to this therapy.

\section{Conclusion}

Our study is most limited by its retrospective nature. As such, during our studied follow-up interval many eyes received adjunctive therapy with alternative intravitreal injections (most commonly anti-VEGF), focal or panretinal photocoagulation, or surgical intervention. Thus, it is somewhat outside of the scope of this study to draw conclusions about the use of DEX as monotherapy. However, we demonstrate that in these patient cohorts the use of repeated DEX treatments over an extended follow-up period improves both functional and anatomic outcomes with limited significant complication risks.

\section{Disclosure}

The authors report no conflicts of interest for this work.

This paper was presented at the 2018 ARVO Annual Meeting as a poster presentation with interim findings. The poster's abstract was published in "Poster Abstracts" in Investigative Ophthalmology \& Visual Sciences: https:// iovs.arvojournals.org/article.aspx ?articleid=2689004.

\section{References}

1. Bakri SJ, Omar AF, Iezzi R, Kapoor KG. Evaluation of multiple dexamethasone intravitreal implants in patients with macular edema associated with retinal vein occlusion. Retina. 2016;36(3):552-557. doi:10.1097/IAE.000000000000750

2. Haller JA, Bandello F, Belfort $\mathrm{R} J r$, et al. Randomized, sham-controlled trial of dexamethasone intravitreal implant in patients with macular edema due to retinal vein occlusion. Ophthalmology. 2010;117(6):1134-1146.e1133. doi:10.1016/j.ophtha.2010.03.032

3. Haller JA, Bandello F, Belfort R Jr, et al. Dexamethasone intravitreal implant in patients with macular edema related to branch or central retinal vein occlusion twelve-month study results. Ophthalmology. 2011;118(12):2453-2460. doi:10.1016/j.ophtha.2011.05.014

4. Li X, Wang N, Liang X, et al. Safety and efficacy of dexamethasone intravitreal implant for treatment of macular edema secondary to retinal vein occlusion in Chinese patients: randomized, sham-controlled, multicenter study. Graefes Arch Clin Exp Ophthalmol. 2018;256(1):59-69. doi:10.1007/s00417-017-3831-6

5. Capone A Jr, Singer MA, Dodwell DG, et al. Efficacy and safety of two or more dexamethasone intravitreal implant injections for treatment of macular edema related to retinal vein occlusion (Shasta study). Retina. 2014;34(2):342-351. doi:10.1097/IAE.0b013e318297f842
6. Hasanreisoglu M, Ozdemir HB, Ozkan K, et al. Intravitreal dexamethasone implant in the treatment of non-infectious uveitis. Turk $J$ Ophthalmol. 2019;49(5):250-257. doi:10.4274/tjo.galenos. 2019.81594

7. Khurana RN, Bansal AS, Chang LK, Palmer JD, Wu C, Wieland MR. Prospective evaluation of a sustained-release dexamethasone intravitreal implant for cystoid macular edema in quiescent uveitis. Retina. 2017;37(9):1692-1699. doi:10.1097/IAE.0000000000001406

8. McCartney M, McCluskey P, Zagora S. Intravitreal dexamethasone implants for non-infectious uveitis. Clin Exp Ophthalmol. 2019;47 (9):1156-1163. doi:10.1111/ceo.13611

9. Pohlmann D, Vom Brocke GA, Winterhalter S, Steurer T, Thees S, Pleyer U. Dexamethasone inserts in noninfectious uveitis: a single-center experience. Ophthalmology. 2018;125(7):1088-1099. doi:10.1016/j.ophtha.2017.12.038

10. Zarranz-Ventura J, Carreno E, Johnston RL, et al. Multicenter study of intravitreal dexamethasone implant in noninfectious uveitis: indications, outcomes, and reinjection frequency. Am J Ophthalmol. 2014;158(6):1136-1145.e1135. doi:10.1016/j.ajo.2014.09.003

11. Boyer DS, Yoon YH, Belfort R Jr, et al. Three-year, randomized, sham-controlled trial of dexamethasone intravitreal implant in patients with diabetic macular edema. Ophthalmology. 2014;121 (10):1904-1914. doi:10.1016/j.ophtha.2014.04.024

12. Castro-Navarro V, Cervera-Taulet E, Navarro-Palop C, MonferrerAdsuara C, Hernandez-Bel L, Montero-Hernandez J. Intravitreal dexamethasone implant Ozurdex(R) in naive and refractory patients with different subtypes of diabetic macular edema. BMC Ophthalmol. 2019;19(1):15. doi:10.1186/s12886-018-1022-9

13. Mello Filho P, Andrade G, Maia A, et al. Effectiveness and safety of intravitreal dexamethasone implant (ozurdex) in patients with diabetic macular edema: a real-world experience. Ophthalmologica. 2019;241(1):9-16. doi:10.1159/000492132

14. Rosenblatt A, Udaondo P, Cunha-Vaz J, et al. A collaborative retrospective study on the efficacy and safety of intravitreal dexamethasone implant (ozurdex) in patients with diabetic macular edema: the european dme registry study. Ophthalmology. 2020;127(3):377-393. doi:10.1016/j.ophtha.2019.10.005

15. Singer MA, Dugel PU, Fine HF, Capone A Jr, Maltman J. Real-world assessment of dexamethasone intravitreal implant in DME: findings of the prospective, multicenter reinforce study. Ophthalmic Surg Lasers Imaging Retina. 2018;49(6):425-435. doi:10.3928/ 23258160-20180601-07

16. Iglicki M, Busch C, Zur D, et al. Dexamethasone implant for diabetic macular edema in naive compared with refractory eyes: the international retina group real-life 24-month multicenter study. The IRGREL-DEX study. Retina. 2019;39(1):44-51. doi:10.1097/ IAE.0000000000002196

17. Bahadorani S, Krambeer C, Wannamaker K, et al. The effects of repeated Ozurdex injections on ocular hypertension. Clin Ophthalmol. 2018;12:639-642. doi:10.2147/OPTH.S148990

18. Monsellato R, Trovato E, Turchetti P, et al. Ocular hypertension management in long-term treatments with intravitreal dexamethasone implants: a 3-year experience. Clin Ter. 2020;170(1):e11-e14.

19. Rajesh B, Zarranz-Ventura J, Fung AT, et al. Safety of 6000 intravitreal dexamethasone implants. Br J Ophthalmol. 2020;104(1):39-46. doi:10.1136/bjophthalmol-2019-313991

20. Schmitz K, Maier M, Clemens CR, et al. Reliability and safety of intravitreal Ozurdex injections. The ZERO study. Ophthalmologe. 2014;111(1):44-52. doi:10.1007/s00347-012-2737-2

21. Malcles A, Dot C, Voirin N, et al. Real-life study in diabetic macular edema treated with dexamethasone implant: the reldex study. Retina. 2017;37(4):753-760. doi:10.1097/IAE.0000000000001234

22. Busch C, Rehak M, Sarvariya C, et al. Long-term visual outcome and its predictors in macular oedema secondary to retinal vein occlusion treated with dexamethasone implant. Br J Ophthalmol. 2019;103 (4):463-468. doi:10.1136/bjophthalmol-2017-311805 
23. Augustin AJ, Kuppermann BD, Lanzetta P, et al. Dexamethasone intravitreal implant in previously treated patients with diabetic macular edema: subgroup analysis of the MEAD study. BMC Ophthalmol. 2015;15(1):150. doi:10.1186/s12886-015-0148-2

24. Bilgic A, Sudhalkar A, Kodjikian L, et al. Pro re nata dexamethasone implant for treatment-naive phakic eyes with diabetic macular edema: a prospective study. Ophthalmol Retina. 2019;3(11):929-937. doi:10.1016/j.oret.2019.05.027

25. Breitbach M, Rack D, Dietzel M, Heinz C, Heiligenhaus A. Efficacy of a dexamethasone implant for the treatment of refractory cystoid macular oedema in non-infectious uveitis. Klin Monbl Augenheilkd. 2016;233(5):601-605.

26. Lin CJ, Chen HS, Su CW, et al. The effect of age and initial central retinal thickness on earlier need of repeat ozurdex treatment for macular edema due to retinal vein occlusion: a retrospective case series. J Ocul Pharmacol Ther. 2017;33(10):763-772. doi:10.1089/ jop. 2017.0070

27. Altunel O, Goktas A, Duru N, Ozkose A, Arifoglu HB, Atas M. The effect of age on dexamethasone intravitreal implant (Ozurdex(R)) response in macular edema secondary to branch retinal vein occlusion. Semin Ophthalmol. 2018;33(2):179-184.

28. Bastakis GG, Dimopoulos D, Stavrakakis A, Pappas G. Long-term efficacy and duration of action of dexamethasone implant, in vitrectomised and non-vitrectomised eyes with persistent diabetic macular oedema. Eye (Lond). 2019;33(3):411-418.

29. Medeiros MD, Alkabes M, Navarro R, Garcia-Arumi J, Mateo C, Corcostegui B. Dexamethasone intravitreal implant in vitrectomized versus nonvitrectomized eyes for treatment of patients with persistent diabetic macular edema. J Ocul Pharmacol Ther. 2014;30 (9):709-716. doi:10.1089/jop.2014.0010

30. Akincioglu D, Kucukevcilioglu M, Durukan AH, Aykas S, Ayyildiz O, Erdurman FC. Outcomes of intravitreal dexamethasone implant in the treatment of recalcitrant diabetic macular edema. Turk J Ophthalmol. 2017;47(5):274-278. doi:10.4274/tjo.28863
31. Malcles A, Dot C, Voirin N, et al. Safety of intravitreal dexamethasone implant (ozurdex): the SAFODEX study. Incidence and risk factors of ocular hypertension. Retina. 2017;37(7):1352-1359. doi:10.1097/IAE.0000000000001369

32. Maturi RK, Pollack A, Uy HS, et al. Intraocular pressure in patients with diabetic macular edema treated with dexamethasone intravitreal implant in the 3-year mead study. Retina. 2016;36(6):1143-1152. doi:10.1097/IAE.0000000000001004

33. Choi W, Park SE, Kang HG, et al. Intraocular pressure change after injection of intravitreal dexamethasone (Ozurdex) implant in Korean patients. Br J Ophthalmol. 2019;103(10):1380-1387. doi:10.1136/ bjophthalmol-2018-312958

34. Asahi MG, Bobarnac Dogaru GL, Onishi SM, Gallemore RP. Strong topical steroid, NSAID, and carbonic anhydrase inhibitor cocktail for treatment of cystoid macular edema. Int Med Case Rep J. 2015;8:305-312. doi:10.2147/IMCRJ.S92794

35. Mirshahi A, Tadayoni R, Mohsenzadeh N, Saeidi Rezvani T, Abrishami M. Efficacy of adjuvant topical timolol-dorzolamide with intravitreal bevacizumab injection in diabetic macular edema: a contralateral eye study. J Curr Ophthalmol. 2019;31(2):168-171.

36. Adan A, Pelegrin L, Rey A, et al. Dexamethasone intravitreal implant for treatment of uveitic persistent cystoid macular edema in vitrectomized patients. Retina. 2013;33(7):1435-1440.

37. Du J, Patrie JT, Prum BE, Netland PA, Shildkrot YE. Effects of intravitreal anti-VEGF therapy on glaucoma-like progression in susceptible eyes. J Glaucoma. 2019;28(12):1035-1040. doi:10.1097/ IJG.0000000000001382
Clinical Ophthalmology

\section{Publish your work in this journal}

Clinical Ophthalmology is an international, peer-reviewed journal covering all subspecialties within ophthalmology. Key topics include: Optometry; Visual science; Pharmacology and drug therapy in eye diseases; Basic Sciences; Primary and Secondary eye care; Patient Safety and Quality of Care Improvements. This journal is indexed on PubMed

\section{Dovepress}

Central and CAS, and is the official journal of The Society of Clinical Ophthalmology (SCO). The manuscript management system is completely online and includes a very quick and fair peer-review system, which is all easy to use. Visit http://www.dovepress.com/ testimonials.php to read real quotes from published authors. 